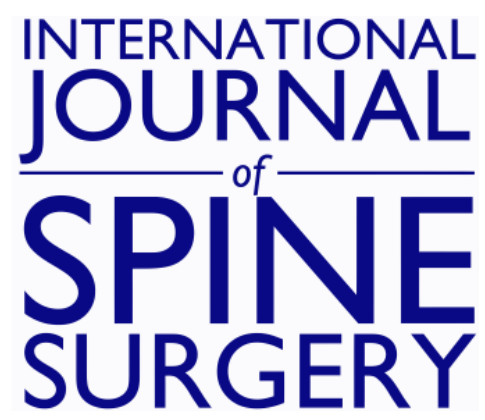

\title{
Long-Term Outcomes Following Lumbar Nucleus Replacement
}

Jeff D. Golan, Frederic Martens, Jeff Griebel, David C. LoPresti, Michael G. Hess and Michael Ahrens

Int J Spine Surg 2021, 15 (6) 1096-1102

doi: https://doi.org/10.14444/8196

http://ijssurgery.com/content/15/6/1096

This information is current as of April 26, 2023.

Email Alerts Receive free email-alerts when new articles cite this article. Sign up at:

http://ijssurgery.com/alerts

The International Journal of Spine Surgery

2397 Waterbury Circle, Suite 1,

Aurora, IL 60504, Phone: +1-630-375-1432 


\title{
Long-Term Outcomes Following Lumbar Nucleus Replacement
}

\author{
JEFF D. GOLAN, MD ${ }^{1}$; FREDERIC MARTENS, MD ${ }^{2}$; JEFF GRIEBEL ${ }^{3}$; DAVID C. LOPRESTI, MD; \\ MICHAEL G. HESS, MD ${ }^{4}$; AND MICHAEL AHRENS, MD \\ ${ }^{1}$ McGill University, Montreal, Canada; ${ }^{2}$ Department of Neurosurgery, Onze-Lieve-Vrouw Hospital, Aalst, Belgium; ${ }^{3}$ Beyond Air Inc, Garden City, New York, USA; \\ ${ }^{4}$ Orthopedic Surgery, London Spine Clinic, London, United Kingdom; ${ }^{5}$ c/o SURGITENT GmbH, Hamburg, Germany
}

\begin{abstract}
Background: Nucleus replacement devices are designed to replace the native pain-generating lumbar nucleus while preserving the annulus fibrosus, endplates, and natural motion. The DASCOR Disc Arthroplasty Device seemed to perform well clinically but was discontinued in 2009 . While there are no commercially available NRDs today, the potential advantages of using such devices have prompted a renewed interest in further developing the technology and assessing long-term outcomes for the DASCOR device.

Methods: A retrospective review of hospital records from a single institution was performed to identify all patients that underwent implantation of a DASCOR device between 2006 and 2009. Clinical outcome and imaging data were gathered to assess device performance of the DASCOR device over an extended period of time. Clinical data were assessed using a visual analog scale for back pain (VAS), Oswestry Disability Index (ODI) scores, and analgesic medication use score (ANS). Magnetic resonance images were systematically evaluated at the index and adjacent disc levels: disc height, Modic changes, Pfirrmann grade, and any implant-associated findings.

Results: Twenty-six patients received DASCOR implants. Follow-up data were available for 22 patients. The mean age at time of implantation was 34.9 years. Long-term data were available for 14 patients (mean, 11.5 years) with a mean improvement in VAS of $4.0+/-2.8(P<0.0001)$, ODI $33.4+/-18.4(P<0.0001)$, and ANS $0.5(P=0.07)$ compared to baseline values, while $46 \%$ developed radiological adjacent segment degeneration on MRI and 54\% did not. In total, 6 patients underwent revision surgery at the index level and 3 underwent surgery at adjacent lumbar levels.

Conclusions: While any conclusions should be interpreted with caution, there was a subgroup of patients that had excellent clinical and radiological outcomes. Additional studies on this device or other similar devices would add to our knowledge regarding ideal treatment of discogenic low back pain in younger patients.

Clinical Relevance: Treatment of discogenic low back pain.

Level of Evidence: 4.
\end{abstract}

Lumbar Spine

Keywords: discogenic, low back pain, nucleus replacement, motion preservation

\section{INTRODUCTION}

Nucleus replacement devices (NRD) are designed to replace the native pain-generating lumbar nucleus while preserving the annulus fibrosus and vertebral endplates. They are intended to re-establish load sharing with the annulus and maintain segmental mobility while preserving disc height. ${ }^{1}$ Early nucleus replacement concepts date back to the late 1950s, even before total disc replacement designs (TDR). ${ }^{2-4}$ An ideal nucleus replacement device will maintain segmental range of motion, provide similar shock-absorbing and cushioning function of a native disc, and reduce adjacent disc degeneration (ADD). In theory, the implantation of such a device would also be possible with less-invasive surgical techniques and tissue disruption when compared to TDR or fusion. Developing such NRD has proven to be extremely challenging for a variety of reasons.

One of the clinical hurdles for NRD has been a high rate of migration and expulsion. ${ }^{5-7}$ One such device, the prosthetic disc nucleus (PDN; Raymedica, Minneapolis, $\mathrm{MN}$ ), was a preformed hydrogel-based device with surgical success rates historically ranging from $77 \%$ to $90 \%{ }^{8}$ The preformed nature of the implant required the creation of an annular flap large enough to deliver the device. Even though the PDN device was implanted in hundreds of patients and demonstrated early surgical success, device migration rates were reported as high as $26 \%{ }^{8}$

Analysis of the PDN outcomes led to the development of another NRD, the DASCOR Disc Arthroplasty Device (Disc Dynamics Inc., Eden Prairie, MN). This 

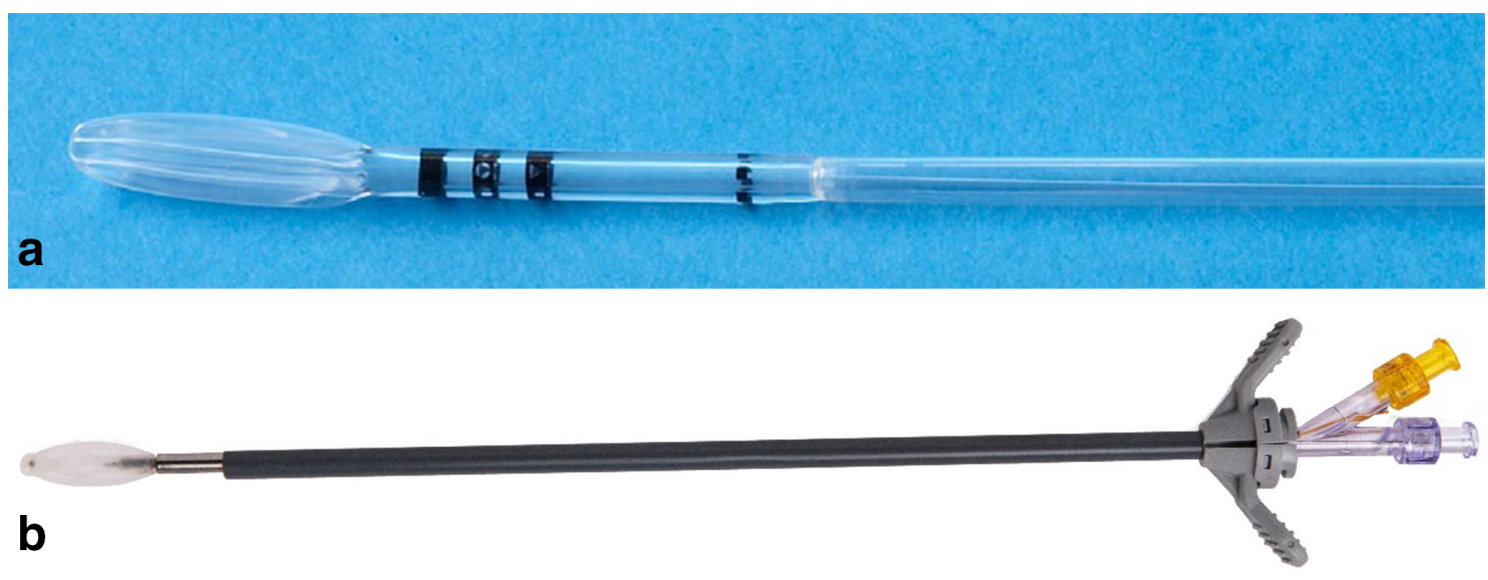

Figure 1. (A) DASCOR delivery catheter and implant. (B) PerQdisc delivery device and implant.

was an in situ cured polyurethane balloon-based device, which resulted in form-fitted, uniquely shaped for the patient's anatomy, and was capable of being delivered through a minimal annular access channel (approximately $5.5 \mathrm{~mm}$ ). The DASCOR device achieved favorable clinical and radiographic results in an interim analysis that reported 2-year outcomes on 85 patients as part of 2 European clinical trials. ${ }^{9}$ The unique design characteristics and implantation techniques resulted in a significant improvement over the PDN device, with no reported incidence of migration or expulsion. Following the clinical trial success of the DASCOR device, a CE mark was granted. It was commercially available until 2009 when the manufacturer ceased operations over concerns regarding the biocompatibility of the uncured polymer. This was only an issue with uncured polymer; once cured, the device was considered biocompatible. The need to redesign the polymer led to financial issues and closure of the company. No long-term follow-up of these patients currently exists.

As of the date of this publication, there is still no NRD available for clinical use. However, 1 is currently in clinical trials, the PerQdisc device (Spinal Stabilization Technologies, Kilkenny, Ireland). The PerQdisc is very similar to the DASCOR device (Figure 1) because they are both form fitting, in situ formed, polymer-filled balloon implants with nearly identical elastic properties and insertion techniques. The main difference is that the PerQdisc uses a room temperature vulcanizing radiopaque silicone polymer with a well-known history of biocompatibility, which also provides better visualization on imaging. These similarities have created renewed interest in the long-term follow-up (LTFU) of DASCOR patients to gauge effectiveness of such NRD with regard to clinical outcomes and preservation of segmental integrity at the index and adjacent levels.
Encouraging long-term data would justify the need to re-explore this technology.

A clinical protocol was developed to address the long-term outcome of the DASCOR device. We initially hoped to follow-up on all 85 patients that were previously published. ${ }^{9}$ Unfortunately, the closure of Disc Dynamics Inc. made it impossible to retrieve all prior DASCOR clinical trial records. We were able to identify 1 clinical trial institution (Onze Lieve Vrouw Ziekenhuis Aalst, Belgium) that was able to complete LTFU for all their patients implanted with the DASCOR device. This individual center series represents the lengthiest follow-up outcomes study on DASCOR patients to date.

\section{METHODS}

\section{Study Design and Patient Selection}

After approval by the institutional review board (Onze Lieve Vrouw Ziekenhuis, Aalst, Belgium), a retrospective review of hospital records was performed to identify all patients that underwent implantation of a DASCOR nucleus replacement device between 2006 and 2009. Inclusion criteria at the time of surgery are listed in (Table 1). Identified patients were contacted to have long-term clinical and radiological follow-up evaluations. Most patients were part of a clinical trial $(63 \%)$, the rest were implanted commercially. Clinical outcome data were gathered using a visual analog scale (VAS) for back pain, Oswestry Disability Index (ODI) scores, and analgesic medication use score.

Radiological outcome was evaluated using new magnetic resonance imaging (MRI) scans. X-rays were not performed to avoid unnecessary radiation exposure, and therefore range of motion assessment was not 
Table 1. Inclusion criteria used for patient enrollment at the time of surgery.

\begin{tabular}{l}
\hline \multicolumn{1}{c}{ Inclusion Criteria } \\
\hline - Age 21 to 70 years \\
- Degenerative disc disease as evidenced by: \\
- History, clinical and radiographic findings \\
- Darkened disc on MRI \\
- Decreased disc height compared to adjacent disc(s) \\
- Positive discography at the affected level \\
- One lumbar spine level condition from L2 to S1 \\
- Minimum disc height of $5.5 \mathrm{~mm}$ \\
- Six monthon of failed nonoperative care \\
- Patient is able to complete a 2-year follow-up visitation schedule \\
- VAS score $\geq 5$ on a 10 -point scale \\
- ODI score $\geq 40$ on a 100-point scale \\
\hline
\end{tabular}

Abbreviations: MRI, magnetic resonance imaging; ODI, Oswestry Disability Index; VAS, visual analog scale.

possible. Baseline, 6-week, 1-year, and LTFU MRIs were all analyzed by an independent core lab (Raylytic GmbH, Leipzig, Germany). The following items were systematically evaluated at the index and adjacent disc levels: disc height (anterior, middle, and posterior measurement points), endplate Modic changes, Pfirrmann grade, and any implant-associated findings (such as device breakage, migrations, or expulsions). ADD was defined as worsening Pfirrmann grade, new Modic changes, loss of disc height exceeding $3 \mathrm{~mm}$, need for secondary surgery at the adjacent level, or new onset spondylolisthesis. Migrations were defined as any portion of the device that was outside of the outer annular margin, and expulsions were defined as any device that was completely outside of the outer annular margin.

\section{Surgical Technique}

All devices in this series were implanted with the patient in a supine position using a standard retroperitoneal anterior approach to gain access to the intervertebral disc for nucleus removal and device implantation. The detailed technique and device description were previously described by Ahrens et al. ${ }^{9}$ In summary, a series of specifically curved rongeurs, each matching a different geometrically mapped region in the nucleus space, was used multiple times in sequence for a systematic total nucleus removal. A DASCOR imaging balloon was then inserted and filled with contrast under fluoroscopy to estimate final implant volume and position, then removed for final implant placement. The DASCOR polyurethane implant balloon was then inserted and filled with polyurethane to create a custom-fit implant. Once cured, the wound was closed in standard fashion.

\section{Outcomes Assessment}

The clinical status of all patients was evaluated by reviewing the individual patient charts at each of the following time points: pre-operatively, post-operatively at 12 months, and then at LTFU. Patients completed patient-reported outcome measure (PROM) questionnaires, including ODI, back VAS, and use of analgesic medication using an analgesic score (ANS) rated on a 0 to 4 point scale with $0=$ no analgesic medication, $1=$ anti-inflammatory medication, 2 = weak opioid analgesics, and 3 and 4 equating to stronger and more frequent use of opioids.

\section{Statistical Methods}

We report the mean and standard deviation for continuous variables including age at surgery, body mass index, follow-up duration, ANS, VAS, and ODI scores, and change in disc height levels. For nominal measures including gender, smoking status, and level treated, we reported in percentages. Clinical outcomes ANS, VAS, and ODI scores were measured repeatedly at baseline and through follow-up. Separate linear mixed models were fit against these outcomes to account for the dependence of repeated measures within a patient population. ${ }^{10}$ In these linear mixed models, clinical outcomes were dependent variables, and a time indicator variable was the independent variable. After model parameters were obtained from the linear mixed models, contrasts were made to estimate changes in these outcomes from baseline measures. $P$ values were also reported for these changes. Analyzes were conducted with SAS software 9.4 (SAS Institute Inc., Cary, NC).

\section{RESULTS}

Twenty-six patients received DASCOR implants between January 2006 and November 2009. Three patients were lost to follow-up and 1 patient had revision surgery due to persistent back pain prior to the 1-year follow-up visit and were excluded from this study leaving a total of $22(85 \%)$ patients for analysis. The patients were divided into 2 groups based on surgical outcome and were also compared to a larger cohort based on the data published by Ahrens. ${ }^{9}$

Eight patients did not wish to undergo long-term clinical follow-up (MRI or questionnaires) but did have clinical data and radiographic images at 1 year following surgery and were labeled as group A. There were 3 male and 5 female patients, with 4 that had surgery at L4/5 and 4 at L5/S1, the mean age at the time of surgery was $38.5( \pm 3.2)$ years. Six of these patients were treated 


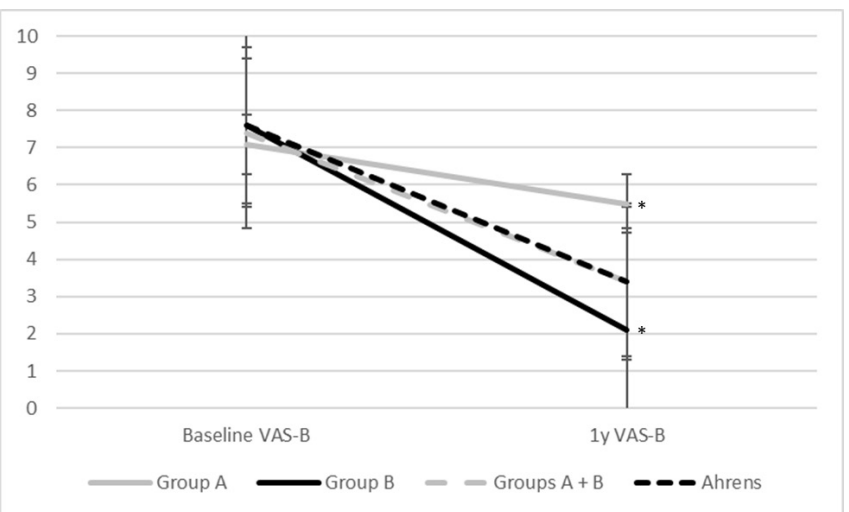

Figure 2.. Change in mean ( \pm SEM) back-visual analog scale (VAS) baseline to 1 year for all groups and the comparison group (Ahrens et $\mathrm{al}^{9}$ ). ${ }^{*}$ Difference between group $\mathrm{A}$ and $\mathrm{B}, P=0.003$.

as part of a clinical trial (DASCOR PM-501) and 2 were treated commercially. While long-term radiographic follow-up is not available, $5 / 8$ patients did not have any migration or expulsion at the 1-year mark (3 patients did not have images available). All patients had baseline and 1-year PROM scores. There was a modest improvement in mean PROM scores at the 1-year follow-up period in 8 patients (Figures 1-3). Ultimately, all 8 patients had secondary surgery. Six patients had revision surgery at the index level with implant removal at a mean of 2.18 years (range $0.77-5.64$ ) from the time of their initial surgery: Five patients had anterior lumbar interbody fusion (ALIF; mean 2.71 years, range 1.02-5.64 years) and 1 had posterior lumbar interbody fusion (at 3.32 years). Two patients did not require revision surgery at the index level but underwent secondary surgery at other lumbar levels: 1 patient had insertion of an interspinous device, while the other underwent posterior dynamic pedicle stabilization.

Fourteen patients had clinical and radiographic data 1 year after surgery, were also willing to undergo

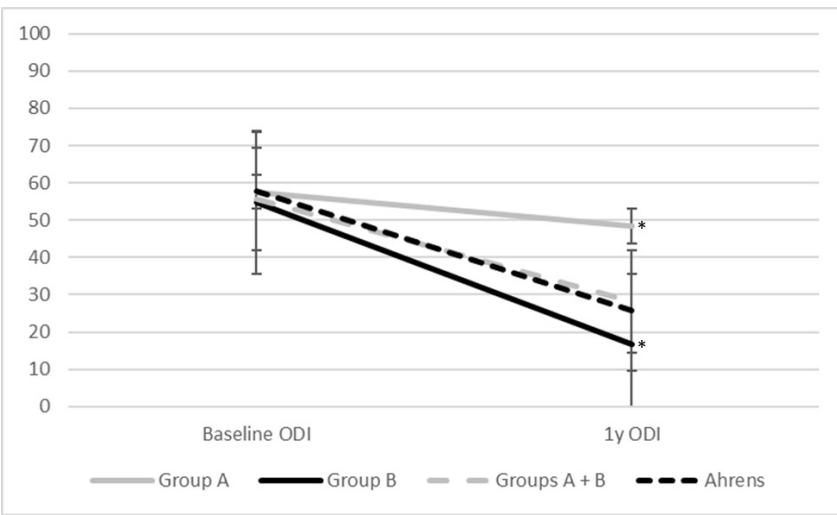

Figure 3.. Mean ( \pm SEM) change in Oswestry Disability Index (ODI) baseline to 1 year for all groups and the comparison group (Ahrens et $\mathrm{al}^{9}$ ). ${ }^{*}$ Difference between group $\mathrm{A}$ and $\mathrm{B}, P=0.001$. long-term clinical follow-up, and were labeled as group B. There were 13 females and 1 male. Nine had surgery at $\mathrm{L} 4 / 5$ and 5 at $\mathrm{L} 5 / \mathrm{S} 1$. The mean age at surgery was $32.6 \pm 2.1$ years. Ten of these patients were treated as part of a clinical trial (DASCOR PM -501) and 4 were treated commercially. Baseline PROM and 1-year follow-up data were available for all 14 patients. LTFU data at mean 11.5 years (range 9.6-12.7) following initial surgery were available for 13 of the 14 patients, with a mean improvement in back VAS of $4.0 \pm 2.8$ $(P<0.0001)$, ODI $33.4 \pm 18.4(P<0.0001)$, and ANS $0.5(P=0.07)$ (Table 2, Figure 4$)$. There were no revision surgeries in this group; 1 patient in this group had secondary surgery at the adjacent level with ALIF at 8 years post implantation. Baseline values for groups $\mathrm{A}$ and B are summarized in Table 3; there were no significant differences in baseline ODI, back VAS, ANS, or age. group B had more women, smokers, and patients treated at the L4/5 level when compared to group A.

Long-term MRIs were performed on all group B patients (Figure 5). One patient could not be adequately assessed due to metallic artifact; there were no expulsions or migrations observed. The mean disc height in 13 of 14 patients (patient 009 had metallic artifact obscuring the disc space) at the index level between the 6 weeks postsurgery and long-term MRI revealed a mean loss of $1.3 \mathrm{~mm}(+0.8-3.1 \mathrm{~mm})$ with $1(8 \%)$ patient having a mean disc height loss of more $3 \mathrm{~mm}$ $(3.1 \mathrm{~mm})$. When comparing the adjacent superior disc level between 6 weeks postsurgery and long-term MRI, there was a mean disc height loss of $0.39 \mathrm{~mm}(+1.1-3.5$ $\mathrm{mm})$, with $1(8 \%)$ patient having a disc height loss of more $3 \mathrm{~mm}(3.5 \mathrm{~mm})$.

Preoperative MRI scans only included T2-weighted images and revealed 2/12 patients with an increased signal at either endplate of the index level (2 patients did not have preop MRI images available for this study). At LTFU, 13 patients had adequate MRIs for evaluating the index level. Of these, 7 patients (54\%) had Type 2 changes and $6(46 \%)$ patients had Type 3 changes. In addition, 1 patient had a new Type 3 change in an adjacent superior disc level, while 1 other patient had new Type 1 change at the inferior disc level.

The Pfirrmann grade classification was applied to baseline and follow-up MRIs. The index level was not evaluated since the native nucleus was removed. The Pfirrmann grade of the adjacent inferior level progressed in 1 patient, and 1 other patient had ALIF surgery at the time of assessment. When a comparison was possible, the Pfirrmann grade of the adjacent superior level progressed in 3 patients, while 1 other patient maintained 
Table 2. Patient-reported outome measures for long-term follow-up patients.

\begin{tabular}{|c|c|c|c|c|c|c|c|}
\hline PROM & Baseline & & 12 Months & & & LTFU & \\
\hline & Mean (SEM) & Mean (SEM) & Mean Change vs Baseline (SEM) & $P$ Value & Mean (SEM) & $\begin{array}{l}\text { Mean Change vs Baseline } \\
\text { (SEM) }\end{array}$ & $P$ Value \\
\hline ODI & $55.1(3.4)$ & $18.5(3.7)$ & $-36.6(19.0)$ & $<0.0001$ & $21.7(4.5)$ & $-33.4(18.4)$ & $<0.0001$ \\
\hline VAS back & $7.6(1.4)$ & $2.2(2.1)$ & $-5.4(2.9)$ & $<0.0001$ & $3.6(2.6)$ & $-4.0(2.8)$ & $<0.0001$ \\
\hline ANS & $1.3(0.9)$ & $0.5(0.6)$ & $-0.8(1.1)$ & 0.002 & $0.8(1.2)$ & $-0.5(1.4)$ & 0.07 \\
\hline
\end{tabular}

Abbreviations: ANS, analgesic score; LTFU, long-term follow-up; ODI, Oswestry Disability Index; PROM, patient-reported outcome measure; VAS, visual analog scale.

the same grade but developed a grade I spondylolisthesis.

Overall, using our definition for ADD, we found 6 patients (46\%) developed ADD while 7 (54\%) did not. No correlation between disc height loss, Modic changes, Pfirrmann grade, and clinical outcomes (ODI/ VAS/ANS) was observed.

\section{DISCUSSION}

Long-term safety concerns of NRD include the risk of expulsion and the response of native tissues to the device. This is particularly relevant as the mean age at the time of implantation in this 22 patient series, operated at a single center, was quite young at 34.9 years. In this long-term study, 2 distinct patient populations were self-defined. Group A consisted of 8 patients that did not wish to fill out PROMs or undergo follow-up MRIs but nonetheless provided valuable long-term data on their ultimate surgical results. Group B consisted of 14 patients that participated fully in our study. When both groups are pooled together, their baseline and 1-year outcomes in ODI, VAS Back, and ANS are near identical to the original published article by Ahrens, ${ }^{9}$ suggesting some of the current findings may be extrapolated to the original larger set of patients. We were able to obtain anonymized patient-level data from the original authors on 74 of the original 85 DASCOR patients with a full

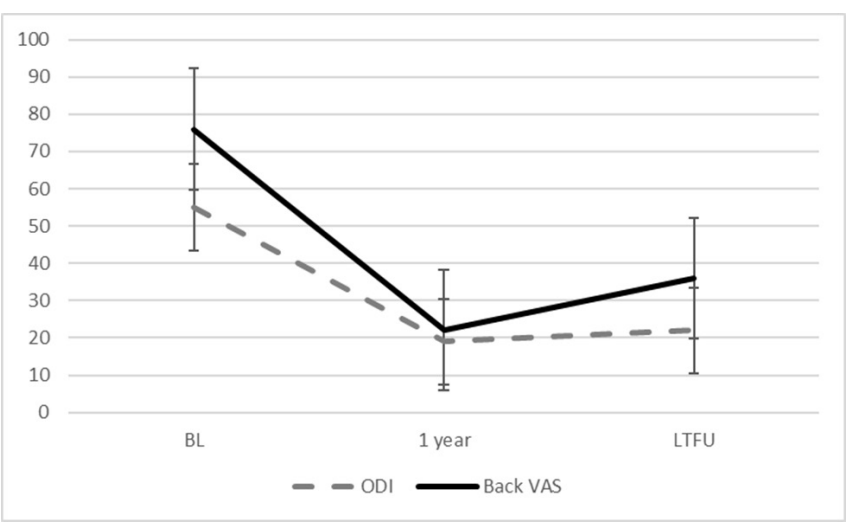

Figure 4. Baseline (BL), 1-year, and long-term follow-up (LTFU) patientreported outcome measures reported as mean \pm SEM. Back visual analog scale (VAS) reported in $\mathrm{mm}$.
PROM data set at 1 year postsurgery for comparison. Groups A and B had comparable PROM scores at baseline but quite divergent outcomes at 1 year with results significantly superior in group B. Six patients $(75 \%)$ in group A needed revision of their implanted DASCOR device, and the other 2 patients $(25 \%)$ needed other lumbar surgeries, while only 1 of 14 patients $(7 \%)$ in group B needed a secondary surgery (ALIF at an adjacent level) 8 years after the initial implant surgery.

The encouraging outcomes in group B are also apparent in their MRI results. There were no expulsions or migrations noted, and a relative paucity of significant adjacent segment degeneration. Six patients $(46 \%)$ developed radiological ADD while 7 (54\%) did not, at a mean of 11.5 years. Subsidence (loss of disc height $\geq 3$ $\mathrm{mm}$ ) occurred in 1 patient at the index level and 1 other patient at an adjacent level. Furunes ${ }^{11}$ et al assessed the 8-year follow-up on 126 of 173 patients from a randomized trial comparing TDR with multidisciplinary rehabilitation using MRI. ADD occurred in $40 \%$ of TDR and $42 \%$ nonoperative patients. There was no association between ADD and change in ODI.

Modic et $\mathrm{al}^{12,13}$ and de Roos et al ${ }^{14}$ described endplate MRI signal intensities associated with degenerative intervertebral disc disease. In this patient population with implantation of the DASCOR device uniformly increased the central endplate stress ${ }^{15}$ and according to Wolff's law, the increased stress would have led to central endplate bone re-formation. ${ }^{16}$ This is supported by long-term radiological data, which show the presence of Modic Type 2 and 3 changes in all patients, representing structural adaptation of the endplate. The clinical relevance of these endplate changes is unclear given that all endplates remained structurally intact, and $87 \%$ of these patients had excellent long-term clinical outcomes.

The separation of patients into groups A and B is heavily biased by each patient's own clinical results. Patients that had better clinical outcome were more likely to participate in this follow-up study, but why did some patients go on to benefit from nucleus replacement more than others? A discrepancy in PROMs was evident as early as 1 year following surgery. There were 
Table 3. Baseline demographics for groups A and B.

\begin{tabular}{|c|c|c|c|}
\hline Characteristic & Group A $(n=8)$ & Group B $(n=14)$ & $P$ Value \\
\hline Age, y, mean (SD) & $38.5(3.2)$ & $32.6(2.1)$ & 0.061 \\
\hline Baseline ODI, mean (SD) & $57.5(4.6)$ & $54.7(3.6)$ & 0.388 \\
\hline Baseline VAS back, mean (SD) & $7.1(0.4)$ & $7.6(0.4)$ & 0.404 \\
\hline Baseline ANS, mean (SD) & $1.3(0.4)$ & $1.3(0.4)$ & 0.357 \\
\hline Sex, M/F, $n(\%)$ & $5(63 \%) / 3(38 \%)$ & $1(7 \%) / 13(93 \%)$ & \\
\hline Smoker, $n(\%)$ & $1(12.5 \%)$ & $6(43 \%)$ & \\
\hline \multicolumn{4}{|l|}{ Level treated, $n(\%)$} \\
\hline $\mathrm{L} 4 / 5$ & $4(50 \%)$ & $9(64 \%)$ & \\
\hline $\mathrm{L} 5 / \mathrm{S} 1$ & $4(50 \%)$ & $5(36 \%)$ & \\
\hline
\end{tabular}

Abbreviations: ANS, analgesic score; ODI, Oswestry Disability Index; VAS, visual analog scale.

demographic differences between the 2 groups, as group A was older by a mean of 6.0 years (not statistically significant), while group B had much higher percentage of female patients (63\% vs 93\%). Another possible difference, considering all 8 patients in group A had secondary surgeries, may be related to the accuracy of diagnosis. The original inclusion criteria by Ahrens ${ }^{9}$ included positive discography at the index level. While this technique was widely considered the Gold standard at the time for diagnosing discogenic back pain, a number of studies have since challenged the accuracy of provocative discography. Current guidelines for performing provocative discography may be associated with better positive predictive value and improved safety profile. ${ }^{17}$ Additionally, Ahrens ${ }^{9}$ excluded patients with advanced facet joint disease but did not oblige other diagnostic requirements for those with mild or moderate facet joint disease nor documented the use of sacroiliac joint provocative maneuvers. It is possible that additional exclusionary tests to rule out other sources of low back pain using validated medial brachial blocks or sacroiliac joint blocks or metabolic imaging, such as bone scan with single photon emission computed tomography, could have further distilled out those more likely to benefit from NRD.

This study has several limitations. The study populations of groups A and B appear to have had similar features to the larger published multicentric population of patients previously published by Ahrens ${ }^{9}$ with 2-year follow-up data, but still represents only 1 center. A larger study would have evened out variabilities noted in demographics. Longer clinical follow-up on group A patients could have been helpful to confirm the trends at 1 year, although 10+ year MRI follow-up would not have made a difference as most of these patients went on to have their implants removed. It is also difficult to comment on the actual cause of failure in group $\mathrm{A}$ without relevant clinical and additional imaging information to assure the implants themselves did not fail or were not otherwise directly related to poor clinical outcomes. At least it is known that none of the DASCOR trial patients had migration or expulsion at 2 years, and none of the patients in group B did, at 11.5 years. Additional limitation, inherent to all retrospective studies, includes some data that were not available for all patients.
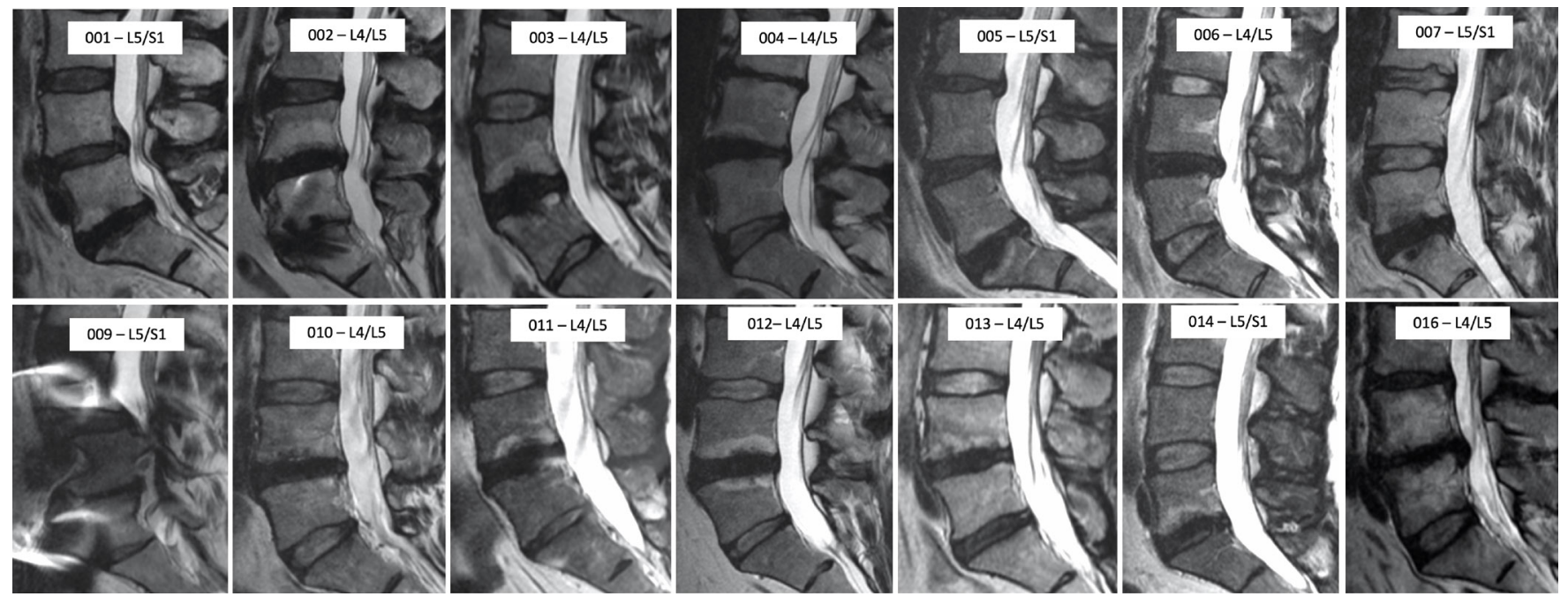

Figure 5. Long-term magnetic resonance imaging (MRI) results, mean 11.5 years. T2-weighted midsagittal views. 
The results of this series of patients support this type of NRD as a viable long-term treatment in well selected patients. It may be worthwhile to revisit the technical limitations that prevented DASCOR from gaining wider acceptance and full regulatory clearance. The need for improving the fundamentals of diagnosing discogenic low back pain is essential in future trial designs.

\section{CONCLUSIONS}

We were able to analyze LTFU data for a subset of patients at a single institution participating in the DASCOR clinical trial. While the clinical and radiological outcomes are very encouraging, this series reports on a minority of patients originally implanted with the DASCOR device and conclusions should be interpreted with caution. Additional studies on this device or other similar devices would add to our knowledge regarding ideal treatment of discogenic low back pain in younger patients. Continued enthusiasm for NRDs appears warranted.

\section{REFERENCES}

1. Reitman CA, Hipp JA, Nguyen L, et al. Changes in segmental intervertebral motion adjacent to cervical arthrodesis: a prospective study. Spine. 2004;29(11):E221-6. doi:10.1097/00007632-200406010-00022

2. BaoQB, Yuan HA. New technologies in spine: nucleus replacement. Spine. 2002;27(11):1245-1247. doi:10.1097/00007632200206010-00020

3. Fernström U. Arthroplasty with intercorporal endoprothesis in herniated disc and in painful disc. Acta Chir Scand Suppl. 1966;357:154-159

4. Szpalski M, Gunzburg R, Mayer M. Spine arthroplasty: a historical review. Eur Spine J. 2002;11 Suppl 2:S65-84. doi:10.1007/ s00586-002-0474-y

5. Rosales-Olivares LM, Perez-Viquez A, MiramontesMartinez V, et al. Experience with prosthetic disc nucleus device in Mexico [Final report of a 4-year follow-up]. Cir Cir. 2007;75:31-36

6. Beaubien BF, Sherman TN, Gullickson AL, Seme SJ. Subsidence characteristics of two nucleus replacement devices compared to a PEEK interbody fusion device. Spine Arthroplasty Society (SAS) 7th Annual Meeting: Global Symposium on Motion Preservation Technology. 2007

7. Schnake KJ, Weigert F, Kandziora F, et al. Local vertebral body destruction after migration of a nucleus replacement. Z Orthop Unfall. 2007;145(5):649-651. doi:10.1055/s-2007-965663

8. Ray CH. Chapter 14 - The Raymedica prosthetic disk nucleus (PDN): stabilizing the degenerated lumbar vertebral segment without fusion or total disc replacement. In: Kim DH, Cammisa FP, Fessler RG, eds. Dynamic Reconstruction of the Smic Reconstruction of the Spine. Thieme Medical Publishers inc; 2006:105-113

9. Ahrens M, Tsantrizos A, Donkersloot P, et al. Nucleus replacement with the DASCOR disc arthroplasty device: interim two-year efficacy and safety results from two prospective, non-randomized multicenter European studies. Spine. 2009;34(13):1376-1384. doi:10.1097/BRS.0b013e3181a3967f

10. Laird NM, Ware JH. Random-effects models for longitudinal data. Biometrics. 1982;38(4):963-974. doi:10.2307/2529876

11. Furunes H, Hellum C, Espeland A, et al. Adjacent disc degeneration after lumbar total disc replacement or nonoperative treatment: a randomized study with 8-year follow-up. Spine. 2018;43(24):1695-1703. doi:10.1097/BRS.0000000000002712

12. Modic MT, Masaryk TJ, Ross JS, et al. Imaging of degenerative disk disease. Radiology. 1988;168(1):177-186. doi:10.1148/ radiology.168.1.3289089

13. Modic MT, Steinberg PM, Ross JS, et al. Degenerative disk disease: assessment of changes in vertebral body marrow with MR imaging. Radiology. 1988;166(1 Pt 1):193-199. doi:10.1148/radiology.166.1.3336678

14. de Roos A, Kressel H, Spritzer C, et al. MR imaging of marrow changes adjacent to end plates in degenerative lumbar disk disease. AJR Am J Roentgenol. 1987;149(3):531-534. doi:10.2214/ ajr.149.3.531

15. Tsantrizos A, Ordway NR, Yuan HA. Segmental flexibility and endplate contact stress of the DASCORTM device. Montreal, Quebec, Canada: Spine Arthroplasty Society; 2006:132

16. Turner $\mathrm{CH}$. Functional determinants of bone structure: beyond Wolff's law of bone transformation. Bone. 1992;13(6):403-409. doi:10.1016/8756-3282(92)90082-8

17. Bogduk N. Practice guidelines for spinal diagnostic and treatment procedures, second edition. San Franciscso, CA: International Spine Intervention Society; 2013

Funding: Funding for this study was provided by Spinal Stabilization Technologies.

Declaration of Conflicting Interests: Jeff Golan, Frederic Martens, David LoPresti, Michael Hess, and Michael Ahrens received honoraria from Spinal Stabilization Technologies. Jeff Griebel is an employee of Spinal Stabilization Technologies. Fredric Martens and Michael Ahrens participated in DASCOR device trials.

Ethics Approval: Institutional Review Board approval: Onze Lieve Vrouw Ziekenhuis, Aalst, Belgium.

Corresponding Author: Jeff D. Golan, 3755 Cote Ste-Catherine Rd, Suite E-929, Montreal, Quebec, H3T 1E2, Canada; jeff.golan@mcgill.ca

Published 20 January 2022

This manuscript is generously published free of charge by ISASS, the International Society for the Advancement of Spine Surgery. Copyright (C) 2022 ISASS. To see more or order reprints or permissions, see http:// ijssurgery.com. 